Sādhanā Vol. 40, Part 2, April 2015, pp. 515-535. (c) Indian Academy of Sciences

\title{
Prediction models for performance and emissions of a dual fuel CI engine using ANFIS
}

\author{
A ADARSH RAI*, P SRINIVASA PAI and B R SHRINIVASA RAO \\ Department of Mechanical Engineering, Nitte Mahalinga Adyanthaya Memorial \\ Institute of Technology, Nitte 574 110, India \\ e-mail: a.adarsh.rai@gmail.com
}

MS received 16 August 2013; revised 6 August 2014; accepted 6 November 2014

\begin{abstract}
Dual fuel engines are being used these days to overcome shortage of fossil fuels and fulfill stringent exhaust gas emission regulations. They have several advantages over conventional diesel engines. In this context, this paper makes use of experimental results obtained from a dual fuel engine for developing models to predict performance and emission parameters. Conventional modelling efforts to understand the relationships between the input and the output variables, requires thermodynamic analysis which is complex and time consuming. As a result, efforts have been made to use artificial intelligence modelling techniques like fuzzy logic, Artificial Neural Network (ANN), Genetic Algorithm (GA), etc. This paper uses a neuro fuzzy modelling technique, Adaptive Neuro Fuzzy Inference System (ANFIS) for developing prediction models for performance and emission parameter of a dual fuel engine. Percentage load, percentage Liquefied Petroleum Gas (LPG) and Injection Timing (IT) have been used as input parameters, whereas output parameters include Brake Specific Energy Consumption (BSEC), Brake Thermal Efficiency (BTE), Exhaust Gas Temperature (EGT) and smoke. In order to further improve the prediction accuracy of the model, GA has been used to optimize ANFIS. GA optimized ANFIS gives higher prediction accuracy of more than $90 \%$ for all parameters except for smoke, where there is a substantial improvement from $46.67 \%$ to $73.33 \%$, when compared to conventional ANFIS model.
\end{abstract}

Keywords. ANFIS; prediction model; genetic algorithm; dual fuel.

\section{Introduction}

The increased cost, shortage of fossil fuels and stringent exhaust gas regulations have aroused interest in the use of dual fuel engines. Most of the early attempts on running the dual fuel engine have been without an adequate basic understanding of the central combustion phenomenon.

${ }^{*}$ For correspondence 
However, it was observed that most of the benefits of the diesel engine could be attained in the dual fuel mode, which includes high thermal efficiency, long operational life and good emission characteristics. It has been found that running a dual fuel engine with turbo-charging will give a similar performance to that of a turbo-charged diesel engine (Jose 2011).

Poonia et al (1999) studied the effects of the intake charge temperature, pilot fuel quantity, throttling of the intake and exhaust gas recirculation on improving the performance of an LPG-Diesel dual-fuel engine. The results indicated that at low outputs, an increase in the intake temperature and pilot quantity improves the BTE. At high outputs, high pilot quantity causes a fall in the efficiency due to rapid combustion. Hydrocarbon (HC) level generally reduces with increase in pilot quantity and intake temperature. Optimum exhaust gas recirculation improves BTE and reduces $\mathrm{HC}$ levels particularly at low and medium outputs. With throttling and exhaust gas recirculation they observed significant reduction in the $\mathrm{HC}$ levels and an improvement in BTE at low loads. Ganesan \& Ramesh (2002) showed that there is an increase in the BTE in the dual-fuel mode at medium and high loads due to a rise in the combustion rate. At low loads, the poor ignition leads to a drop in the BTE and increase in HC and carbon monoxide (CO) levels. Smoke and particulate levels decreased drastically in dual fuel mode. The unburnt HC and CO levels rose at all conditions as compared to diesel operation.

Over the last few decades, there is a sharp increase in vehicle production and transport volume. Environmental concerns such as global warming, greenhouse gas effects, acid rain, and air pollution problems related to the emissions of carbon dioxide $\left(\mathrm{CO}_{2}\right)$, nitrogen oxides (NOx), $\mathrm{CO}$, and unburnt $\mathrm{HC}$, together with the consumption of fossil fuels, combine to create serious problems at a global level. The exhaust emission regulatory bodies around the world have been simultaneously reducing the admissible limits on exhaust emissions over the last decades, while the market has also been striving to maintain or even reduce fuel consumption. In order to comply with these regulations, the automobile industry has undergone significant technological developments in the last few years, creating a high number of new strategies such as electronic control, new injection systems allowing higher pressures, different injection events, etc. As a result, the problem of optimizing the engine management in order to simultaneously comply with emission regulations and fuel economy requirements has become a difficult task, especially due to the increased number of degrees of freedom in the engine operating parameters. This requirement can be met either by conducting comprehensive tests or by modelling the engine operations. Testing the engine under all possible operating conditions and fuel cases are both time consuming and expensive (José et al 2007). On the other hand, developing an accurate model for the operation of CI engine fuelled with different percentages of LPG is too difficult due to the complex nature of the processes involved. As an alternative, engine performance and exhaust emissions can be modelled by using Artificial Intelligence (AI) modelling techniques like Fuzzy Logic, ANN, GA, etc. These new modelling techniques can be applied to estimate the desired output parameters when enough experimental data is provided (Jose 2011).

The work in this paper is an extension of the work carried out in (Rai et al 2012). In the previous work, the focus was on comparing conventional fuzzy logic based model with ANFIS Model, which is a hybrid technique involving fuzzy logic and ANN. The current work tries to further improve the performance of ANFIS model by using GA to optimize the type and number of membership functions of input used in ANFIS Modelling. Percentage load, Percentage LPG and IT are used as input parameters and output parameters include BSEC, BTE and emission parameters include EGT and smoke. The performance of ANFIS has been compared with that of GA optimized ANFIS. 
Table 1. Specifications of the measuring instruments with their performance accuracy.

\begin{tabular}{ll}
\hline Measuring instrument & \multicolumn{1}{c}{ Performance accuracy } \\
\hline Strain gauge load cell & range $0-50 \mathrm{Kg}$. \\
Temperature- $\mathrm{Cr}-\mathrm{Al}$ thermocouple & Range $0-1000^{\circ} \mathrm{C}$. \\
Pressure sensor & Maximum Pressure: $15,000 \mathrm{psi}$. \\
& Resolution : $0.1 \mathrm{psi}$ \\
Smoke meter & Measuring range: $0-100 \%$ opacity in $\%$. \\
& $0-\infty$ absorption $\mathrm{m}^{-1}$. \\
\hline
\end{tabular}

\section{Experimental set-up}

Data from the experiments conducted in a LPG-Diesel CI Engine test rig by Bailkeri (2005) has been used for building the models in the present study. The LPG cylinder is connected to the inlet manifold through a rubber hose provided with a control valve for the induction of LPG along with air during the suction stroke. The rate of diesel injection is controlled by the governor depending on the total energy requirement at different loads in the presence of LPG. The volumetric LPG flow is measured by a gas flow meter. The engine was run on a dual fuel mode at a constant speed of $1500 \mathrm{rpm}$. An injection timing of $27^{\circ}$ before Top Dead Center (bTDC) was used for best performance as specified by the manufacturer (Kirloskar TV -1). The engine was run at a specified load with various LPG/Diesel ratios. At each ratio, performance parameters namely BSEC (kJ/kWh), BTE (\%) and emission parameters namely EGT ( ${ }^{\circ} \mathrm{C}$ ) and smoke levels (HSU - Hartridge Smoke Unit) were measured under steady state conditions. Table 1 shows the performance accuracy of the measuring instruments used for measuring the performance and emission parameters of the engine. The experiments were repeated for various loads viz., $0 \%$, $25 \%, 50 \%, 75 \%$ and $100 \%$ of the full load capacity, and with advanced and retarded injection timings (Rai et al 2012).

\section{Adaptive neuro fuzzy inference system (ANFIS)}

\subsection{Introduction}

An ANFIS is a combination of neural network and fuzzy systems in such a way that neural network is used to determine the parameters of fuzzy systems. ANFIS largely removes the requirement for manual optimization of the fuzzy system parameters. A neural network is used to automatically tune the system parameters, for example the membership function bounds, leading to improved performance without operator intervention. The neuro fuzzy system with the learning capability of neural network and with the advantages of the rule-base fuzzy system can improve the performance significantly and can provide a mechanism to incorporate past observations into the classification process. In neural network, the training essentially builds the system. However, using a neuro fuzzy scheme, the system is built by fuzzy logic definitions and is then refined using neural network training algorithms (Mohd 2008).

Lee et al (2007) showed that fuel spray penetration for a diesel engine can be modelled using fuzzy and ANFIS techniques. The inputs for the model were in-cylinder pressure and air density. Both models were validated by comparing the predicted results against the experimental data. 
The results showed that performance of ANFIS was better than conventional fuzzy, by comparing the correlation coefficients estimated by both the models. Al-Hinti et al (2009) used ANFIS modelling technique for predicting the performance characteristics of a diesel engine. Experimental data sets were divided into training and validation. The training data sets were used to train ANFIS toolbox of MATLAB to model the performance characteristics of diesel engine. Validation data sets that were not used for ANFIS training were used to validate the developed models. The results showed that the ANFIS can be efficiently used to predict the diesel engine performance characteristics.

Literature review reveals that there have been limited efforts made in using ANFIS, which is a hybrid system based on neural networks and fuzzy logic for predicting performance and emission parameters in a dual fuel engine.

\subsection{ANFIS architecture}

Since there are many different neural networks and types of fuzzy logic, there are also many types of neuro-fuzzy implementations. ANFIS is one implementation that has gained some pop-

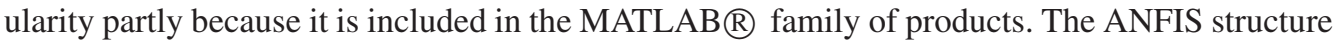

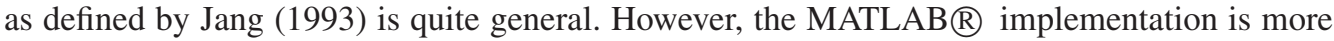

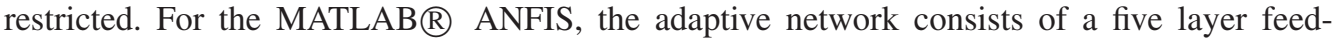
forward network as shown in figure 1, all of the links in the network have unit weights, so the network is only changed through changes to the connections and the functions inside the nodes.

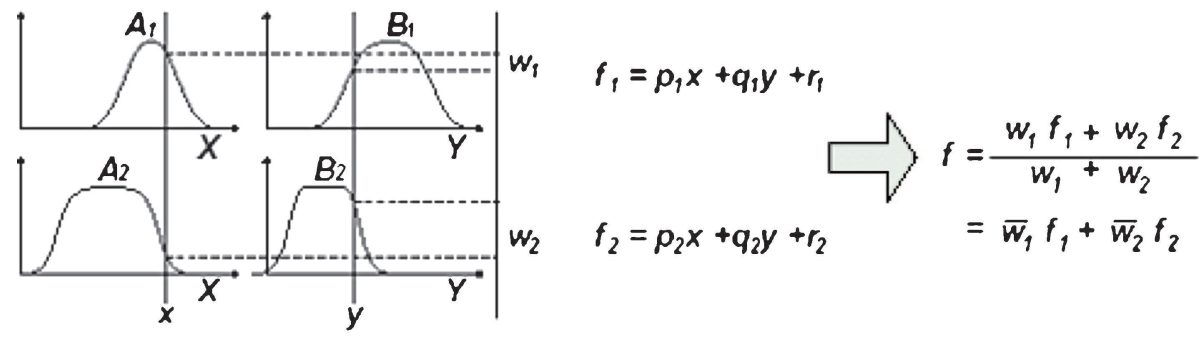

(a)

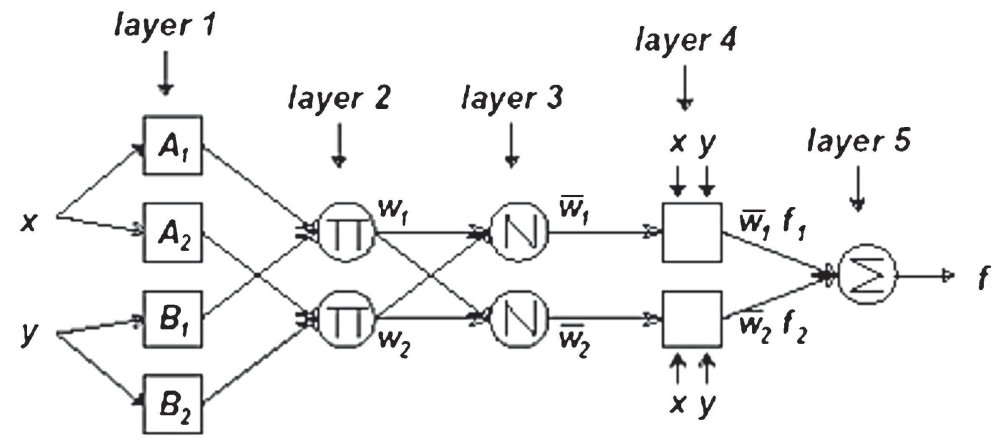

(b)

Figure 1. (a) A two-input first-order Sugeno fuzzy model with two rules; (b) equivalent ANFIS architecture (Sugeno \& Takagi 1983). 
Layer 1: Every node $i$ in this layer is an adaptive node with a node function

$$
\begin{aligned}
& O_{1, i}=\mu_{A_{i}}(x), \text { for } i=1,2, \text { or } \\
& O_{1, i}=\mu_{B_{i-2}}(y) \text { for } i=3,4,
\end{aligned}
$$

where $x$ (or $y$ ) is the input to node $i$ and $A_{i}$ (or $B_{i-2}$ ) is a linguistic label (such as 'small' or 'large') associated with this node. In other words, $O_{1, i}$ is the membership grade of a fuzzy set $A\left(=A_{1}, A_{2}, B_{1}\right.$ or $\left.B_{2}\right)$ and it specifies the degree to which the given input $x$ (or $y$ ) satisfies the quantifier $A$.

Here the membership function for A can be any appropriate parameterized membership function, such as the generalized bell function

$$
\mu_{A_{i}}(x)=\frac{1}{1+\left|\frac{x-c_{i}}{a_{i}}\right|^{2 b_{i}}},
$$

where $\left\{a_{i}, b_{i}, c_{i}\right\}$ is the parameter set. As the values of these parameters change, the bell-shaped function varies accordingly, thus exhibiting various forms of membership functions for fuzzy set A. Parameters in this layer are referred to as premise parameters.

Layer 2: Every node in this layer is a fixed node labelled $\Pi$, whose output is the product of all the incoming signals:

$$
O_{2, i}=w_{i}=\mu_{A_{i}}(x) \mu_{B_{i}}(y), i=1,2 .
$$

Each node output represents the firing strength of a rule. In general, any other T-norm operators that perform fuzzy AND can be used as the node function in this layer.

Layer 3: Every node in this layer is a fixed node labelled N. The $i^{\text {th }}$ node calculates the ratio of the $i^{\text {th }}$ rule's firing strength to the sum of all rules firing strengths:

$$
O_{3, i}=\bar{w}_{i}=\frac{w_{i}}{w_{1}+w_{2}}, i=1,2
$$

For convenience, outputs of this layer are called normalized firing strengths.

Layer 4: Every node $i$ in this layer is an adaptive node with a node function

$$
O_{4, i}=\bar{w}_{i} f_{i}=\bar{w}_{i}\left(p_{i} x+q_{i} y+r_{i}\right),
$$

where $\bar{w}_{\mathrm{i}}$ is a normalized firing strength from layer 3 and $\left\{p_{i}, q_{i}, r_{i}\right\}$ is the parameter set of this node. Parameters in this layer are referred to as consequent parameters.

Layer 5: The single node in this layer is a fixed node labelled $\sum$, which computes the overall output as the summation of all incoming signals (Jang et al 1997)

$$
\text { overall output }=O_{5,1}=\sum_{i} \bar{w}_{i} f_{i}=\frac{\sum_{i} w_{i} f_{i}}{\sum_{i} w_{i}}
$$

\subsection{Learning Algorithm of ANFIS}

The task of the learning algorithm for this architecture is to tune all the modifiable parameters, namely $\left\{a_{i}, b_{i}, c_{i}\right\}$ and $\left\{p_{i}, q_{i}, r_{i}\right\}$, to make the ANFIS output match the training data. When 
Table 2. Two passes in the hybrid learning procedure for ANFIS.

\begin{tabular}{lll}
\hline & \multicolumn{1}{c}{ Forward pass } & Backward pass \\
\hline Premise parameters & Fixed & Gradient descent \\
Consequent parameters & Least-squares estimator & Fixed \\
Signals & Node outputs & Error signals \\
\hline
\end{tabular}

the premise parameters $a_{i}, b_{i}$ and $c_{i}$ of the membership functions are fixed, the outputs of the ANFIS model can be written as

$$
f=\frac{w_{1}}{w_{1}+w_{2}} f_{1}+\frac{w_{2}}{w_{1}+w_{2}} f_{2} .
$$

Substituting Eq. (3) into (6) yields

$$
f=\bar{w}_{1} f_{1}+\bar{w}_{2} f_{2} \text {. }
$$

Substituting the fuzzy if-then rules into Eq. (7), it becomes

$$
f=\bar{w}_{1}\left(p_{1} x+q_{1} y+r_{1}\right)+\bar{w}_{2}\left(p_{2} x+q_{2} y+r_{2}\right) .
$$

After rearrangement, the output can be expressed as

$$
f=\left(\bar{w}_{1} x\right) p_{1}+\left(\bar{w}_{1} y\right) q_{1}+\left(\bar{w}_{1}\right) r_{1}+\left(\bar{w}_{2} x\right) p_{2}+\left(\bar{w}_{2} y\right) q_{2}+\left(\bar{w}_{2}\right) r_{2},
$$

which is a linear combination of the modifiable consequent parameters $p_{1}, q_{1}, r_{1}, p_{2}, q_{2}$ and $r_{2}$. The least squares method can be used to identify the optimal values of these parameters easily. When the premise parameters are not fixed, the search space becomes larger and the convergence of the training becomes slower. A hybrid algorithm combining the least squares method and the gradient descent method is adopted to solve this problem. The hybrid algorithm is composed of a forward and a backward pass. The least squares method (forward pass) is used to optimize the consequent parameters with the premise parameters fixed. Once the optimal consequent parameters are found, the backward pass starts immediately. The gradient descent method (backward pass) is used to adjust optimally the premise parameters corresponding to the fuzzy sets in the input domain. The output of the ANFIS is calculated by employing the consequent parameters found in the forward pass. Table 2 summarizes the activities in each pass. The output error is used to adapt the premise parameters by means of a standard back propagation algorithm. It has been proven that this hybrid algorithm is highly efficient in training the ANFIS (Mohd 2008).

\subsection{ANFIS modelling}

Figure 2 shows the ANFIS training and modelling process, a total of 60 sets of data were selected randomly from 75 sets obtained during experimentation, for ANFIS training. The remaining 15 sets of data were used for testing the trained model, in order to verify its accuracy. Training data is loaded into the MATLAB program and the following membership functions $\{\operatorname{trimf,trapmf,gaussmf,gauss} 2 m f$, pimf,dsigmf, gbellmf, psigmf $\}$ are used for input parameters and $\{$ linear $\}$ for output parameter. The input data were analysed using three classes. 


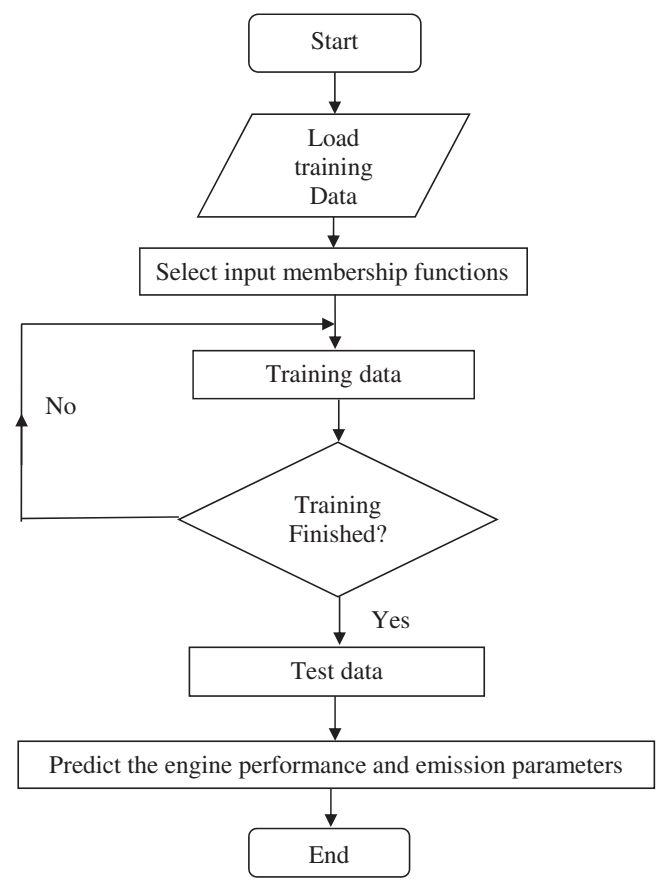

Figure 2. ANFIS training and modelling process.

These eight membership functions are used for all the input parameters (three) in different combinations, and hence $\left(8^{3}=512\right)$ combinations are produced. A fuzzy inference system is created using the above training data and membership functions using an inbuilt function named GENFIS1. The inputs of test data are given to the ANFIS model and output predicted from the

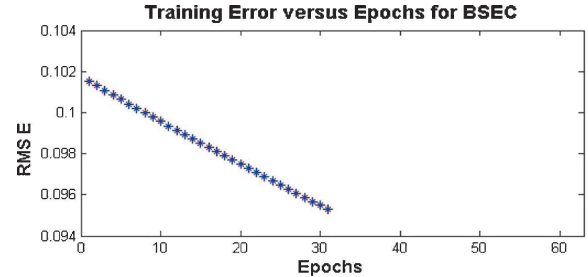

(a)

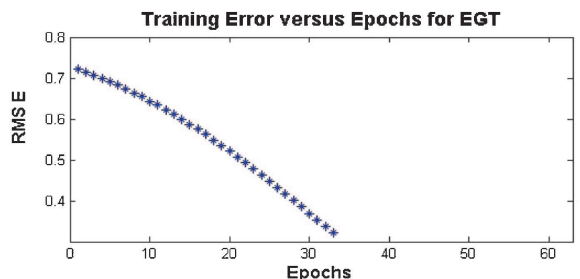

(c)

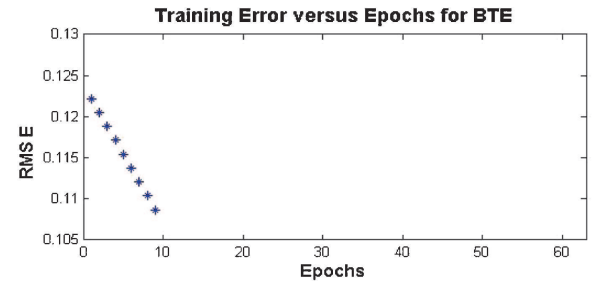

(b)

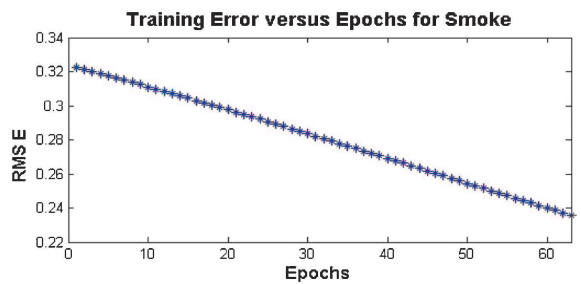

(d)

Figure 3. Training curves of ANFIS prediction model for (a) BSEC, (b) BTE, (c) EGT and (d) Smoke. 
Table 3. Two passes in the hybrid learning procedure for ANFIS.

\begin{tabular}{|c|c|c|c|c|c|c|c|c|c|c|c|}
\hline \multicolumn{6}{|l|}{ BSEC } & \multicolumn{6}{|c|}{ BTE } \\
\hline & No. & Training & MF & MF & MF & & No. & Training & MF & MF & MF \\
\hline Sl & of & Error & Type & Type & Type & Sl & of & Error & Type & Type & Type \\
\hline No & Epochs & (RMSE) & (\%Load) & (I T) & (\% LPG) & No & Epochs & (RMSE) & (\%Load) & (I T) & (\% LPG) \\
\hline 1 & 29 & 0.096 & trimf & trimf & trimf & 1 & 29 & 0.115 & trimf & trimf & trimf \\
\hline 2 & 30 & 0.096 & trimf & gaussmf & trimf & 2 & 17 & 0.109 & trimf & trimf & trapmf \\
\hline 3 & 27 & 0.096 & trimf & gauss $2 \mathrm{mf}$ & trimf & 3 & 32 & 0.114 & trimf & gaussmf & trimf \\
\hline 4 & 29 & 0.096 & gauss2mf & trimf & trimf & 4 & 15 & 0.110 & trimf & gauss $2 \mathrm{mf}$ & trapmf \\
\hline 5 & 27 & 0.096 & trapmf & trapmf & trimf & 5 & 14 & 0.110 & trimf & trapmf & trapmf \\
\hline 6 & 31 & 0.095 & trapmf & gbellmf & $\operatorname{trimf}$ & 6 & 15 & 0.110 & trimf & dsigmf & trapmf \\
\hline 7 & 27 & 0.096 & dsigmf & gaussmf & trimf & 7 & 18 & 0.107 & trimf & gbellmf & trapmf \\
\hline 8 & 27 & 0.096 & dsigmf & dsigmf & trimf & 8 & 14 & 0.110 & trimf & pimf & trapmf \\
\hline 9 & 29 & 0.096 & pimf & trimf & trimf & 9 & 11 & 0.114 & gaussmf & gbellmf & gaussmf \\
\hline 10 & 30 & 0.095 & pimf & gaussmf & trimf & 10 & 9 & 0.106 & gauss $2 m f$ & gbellmf & psigmf \\
\hline \multicolumn{6}{|l|}{ EGT } & \multicolumn{6}{|c|}{ Smoke } \\
\hline & No. & Training & MF & MF & MF & & No. & Training & MF & MF & MF \\
\hline S1 & of & Error & Type & Type & Type & Sl & of & Error & Type & Type & Type \\
\hline No & Epochs & (RMSE) & (\%Load) & (I T) & (\% LPG) & No & Epochs & (RMSE) & (\%Load) & (I T) & (\% LPG) \\
\hline 1 & 33 & 0.307 & trimf & trimf & trimf & 1 & 63 & 0.234 & trimf & trimf & gbellmf \\
\hline 2 & 31 & 0.338 & trimf & gaussmf & trimf & 2 & 36 & 0.292 & trimf & gaussmf & gaussmf \\
\hline 3 & 11 & 0.390 & trimf & dsigmf & gaussmf & 3 & 25 & 0.289 & trimf & gaussmf & gbellmf \\
\hline 4 & 9 & 0.462 & trimf & gbellmf & gaussmf & 4 & 39 & 0.288 & trimf & gbellmf & gaussmf \\
\hline 5 & 8 & 0.460 & trimf & trimf & pimf & 5 & 28 & 0.285 & trimf & gbellmf & gbellmf \\
\hline 6 & 4 & 0.470 & trimf & gaussmf & gauss $2 \mathrm{mf}$ & 6 & 23 & 0.305 & gauss $2 \mathrm{mf}$ & gaussmf & gaussmf \\
\hline 7 & 33 & 0.306 & gauss $2 m f$ & trimf & trimf & 7 & 23 & 0.292 & gauss $2 \mathrm{mf}$ & gaussmf & gbellmf \\
\hline 8 & 7 & 0.479 & pimf & trimf & psigmf & 8 & 24 & 0.304 & gauss $2 \mathrm{mf}$ & gbellmf & gaussmf \\
\hline 9 & 11 & 0.477 & pimf & gbellmf & trapmf & 9 & 23 & 0.292 & gauss $2 \mathrm{mf}$ & gbellmf & gbellmf \\
\hline 10 & 10 & 0.425 & psigmf & trimf & gaussmf & 10 & 27 & 0.286 & pimf & gaussmf & gbellmf \\
\hline
\end{tabular}


model is compared with experimental data using Mean Relative Error (MRE) which is given by

$$
M R E=\left|100 * \frac{\left(t_{j}-o_{j}\right)}{t_{j}}\right|,
$$

where $t_{j}=$ experimental value and $o_{j} \quad=$ predicted value, respectively and if the error is least in that iteration it is noted down and the cycle repeats (Rai et al 2012).

\subsection{Results and discussion}

It is observed that the Root Mean Square Error (RMSE) goes on decreasing with increased number of epochs. The results shown here are for the best possible combination of membership functions selected for the input parameters. There is a variation in terms of number of epochs for different output parameters, with smoke taking maximum number of epochs. The variations of RMSE with number of epochs have been presented for all the output parameters which are shown in figures $3 \mathrm{a}$ to $3 \mathrm{~d}$ (Rai et al 2012).

Table 3 gives optimal membership functions, that were selected from among 512 different combinations studied, for different output parameters.

It is clear from the table that for BSEC with $\{$ trapmf, gbellmfandtrimf $\}$ for input parameters the error was 0.09509 for 31 epochs, for BTE with \{gauss $2 m f$, gbellmfandpsigmf \}, the error was 0.10695 with 9 training epoch, for EGT with $\{g a u s s 2 m f$, trimfandtrimf $\}$, the
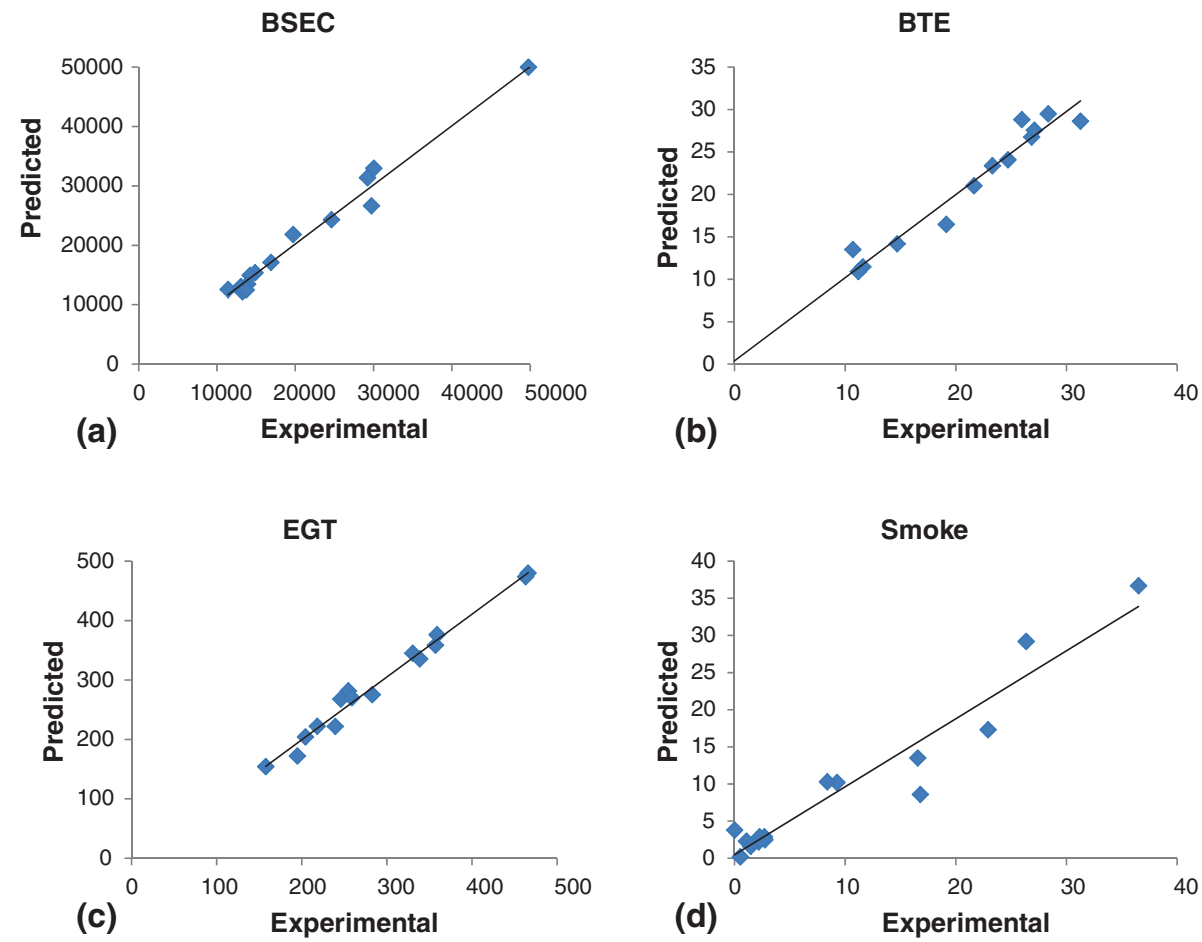

Figure 4. ANFIS predicted versus experimental values for (a) BSEC, (b) BTE, (c) EGT and (d) Smoke. 
Table 4. $\mathrm{R}^{2}$ and prediction accuracy value for ANFIS model.

\begin{tabular}{lcc}
\hline Parameters & $\mathrm{R}^{2}$ & Prediction accuracy on test data (\%) \\
\hline BSEC & 0.986 & 93.33 \\
BTE & 0.977 & 86.67 \\
EGT & 0.983 & 93.33 \\
Smoke & 0.925 & 46.67 \\
\hline
\end{tabular}

error was 0.30566 with 33 epochs and for smoke with $\{$ trimf,trimfandgbellmf $\}$, the error was 0.23436 with 63 epochs. The output parameters studied here required different combination of membership functions for input parameters to achieve a desired level of performance. Also it is clear that for smoke the error was higher with training requiring more time. This has been attributed to noisy data, during the measurement process of smoke (Rai et al 2012).

The ANFIS predictions of the performance and emission parameters for the tested dual fuel engine yielded a good statistical performance. Comparisons of the experimental results and the ANFIS predictions demonstrated that LPG-diesel dual fuel engine can be accurately simulated using ANFIS. Figures 4a-d show ANFIS predicted versus experimental values for BSEC, BTE, EGT and Smoke, respectively. The $\mathrm{R}^{2}$ (coefficient of determination) value and prediction accuracy on test data are given in table 4. It is clear from the table that except for smoke the values of $\mathrm{R}^{2}$ are above 0.95 indicating a high level of correlation and prediction accuracies above $85 \%$ establishing the feasibility of ANFIS based model for predicting performance and emissions of dual fuel engine (Rai et al 2012).

\section{Genetic algorithm}

\subsection{Introduction}

GA is used to give the solution by simulating the evolutionary processes of survival of the fittest, which ensures that the best members of population are retained. The algorithm begins with a set of solutions (chromosomes) called population. Solutions from one population are reproduced to create a new generation of population. Mutations also occurred randomly in each population.

The GA has following features:

- Generates an initial population of random chromosomes.

- Calculates fitness of each chromosome.

- Reproduces next generation by selecting pairs of parents. During reproduction, crossover of the genes and random mutation in some of the children occur.

- The reproduction continues until the best solution is created.

Randomness plays a central role in the GA process; many random procedures are used during execution of GA (Hong-Hee et al 2007). These steps are shown in figure 5.

Piccolo (2001) used GA to energy flow management parameters of an hybrid electric vehicle, which uses electrical motor and internal combustion engine to give high efficiency and low emissions of the vehicle. The flow management parameters like highest desired battery state of charge, lowest desired battery state of charge, etc. were optimized using GA and conventional gradient based procedure and found that GA can be used for optimizing the hybrid electric vehicles. Park (2012) used dimethyl ether (DME) as an alternative fuel to diesel. The design parameters used were in-cylinder flow and combustion process, using a KIVA code; coupled with 




Figure 5. Flow chart for GA.

the Chemkin chemistry solver. The design process was optimized using micro-genetic algorithm. The optimized design for the DME engine showed significant improvement in combustion and emission characteristics, which was compared with conventional diesel engine.

\subsection{GA optimized ANFIS}

In the present work, the GA is used to choose the optimal number of membership functions as well as the type. The number of membership function analysed here includes three, four, five and six. The fitness function of GA is defined as the average of RMSE of ANFIS and number of misclassifications of the ANFIS model. While ANFIS is applied for optimization of the premise parameters (input membership functions) and the consequent parameters (output membership functions), GA will search for the best number of classifications, type of input membership functions and type of output membership functions, such that the fitness function value is minimized. The set of possible input membership functions are $\{$ trimf, trapmf, gbellmf, gaussmf, gauss $2 m f$, pimf, psigmf, dsigmf $\}$, output membership functions are $\left\{\right.$ linear $\left.\left(z=p_{i} X+q_{i} Y+r_{i}\right)\right\}$, where $p_{i}, q_{i}$, and $r_{i}$ are consequent parameters, the number of membership functions for each input is in the range from 3 to 6. (Rai Adarsh 2012).

Figure 6 shows the flowchart for optimizing ANFIS Model using GA. A random population of 20 chromosomes has been initialized and its fitness function is evaluated. The chromosomes are sorted according to the minimum value of the fitness function and then heuristic crossover 


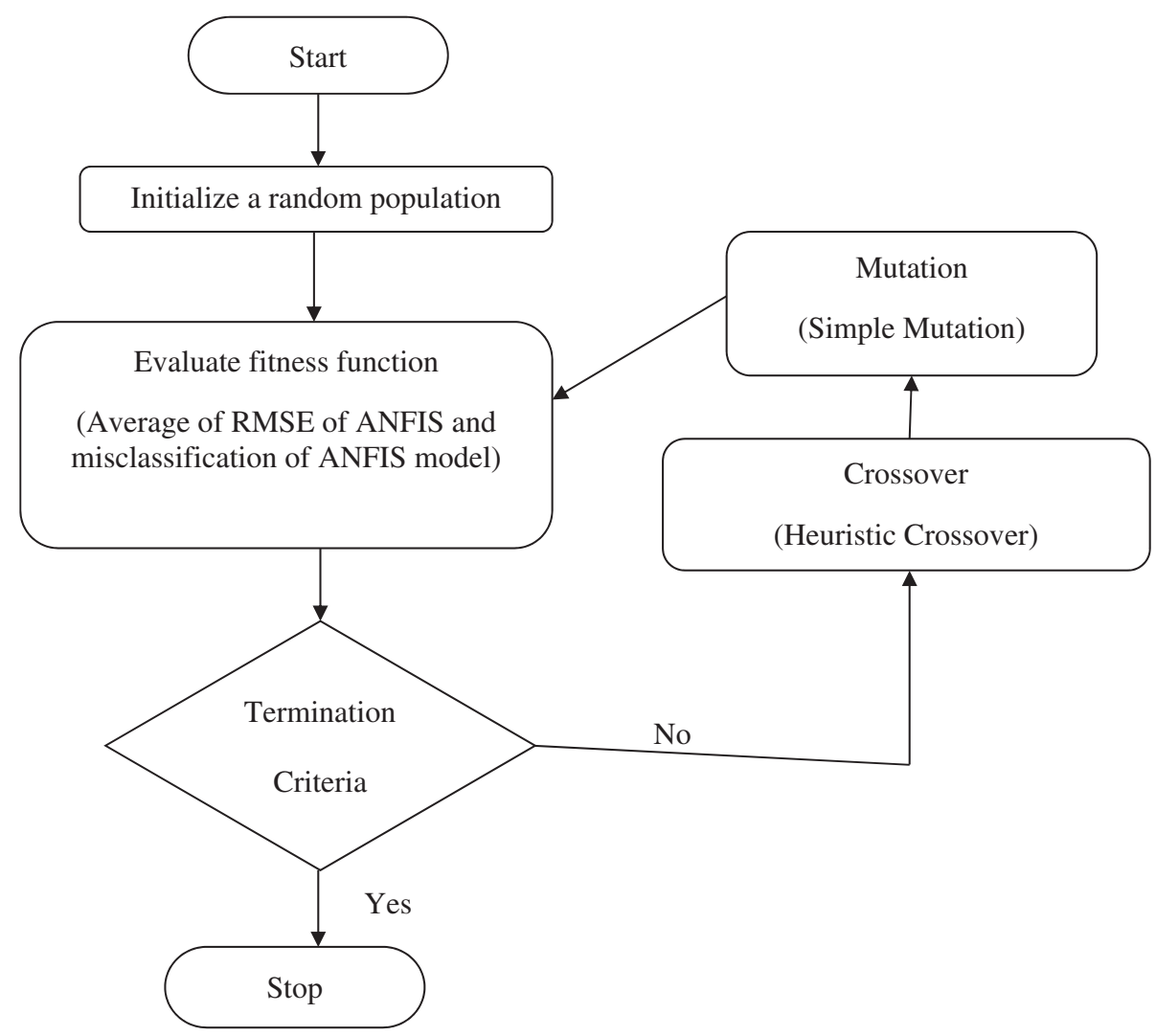

Figure 6. Flowchart for GA optimized ANFIS.

has been applied. Since heuristic crossover helps the chromosomes to move towards the minimum fitness value faster, when compared to other crossover techniques. Mutation functions like non-uniform, uniform and simple mutation are tested and simple mutation has been found to be giving better results. In simple mutation, random values are generated within the higher and lower permissible values and are used to replace the genes of present chromosome. The termination criteria for GA optimization process are either the value of the fitness function which is zero or maximum of 100 generations. (Rai Adarsh 2012).

\subsection{Results and discussion}

Trials have been conducted with 20,30, 40 and 50 chromosomes, but it has been found that for 20 chromosomes, the computational effort is less and there is not much difference in the final results obtained. GA used 100 generations to search for optimal ANFIS configuration. The

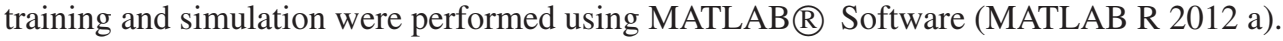

It can be seen that GA helps in finding the optimal number of classes, shape of the membership function for inputs, such that the ANFIS model obtained gives the highest possible prediction accuracy (Rai Adarsh 2012). 

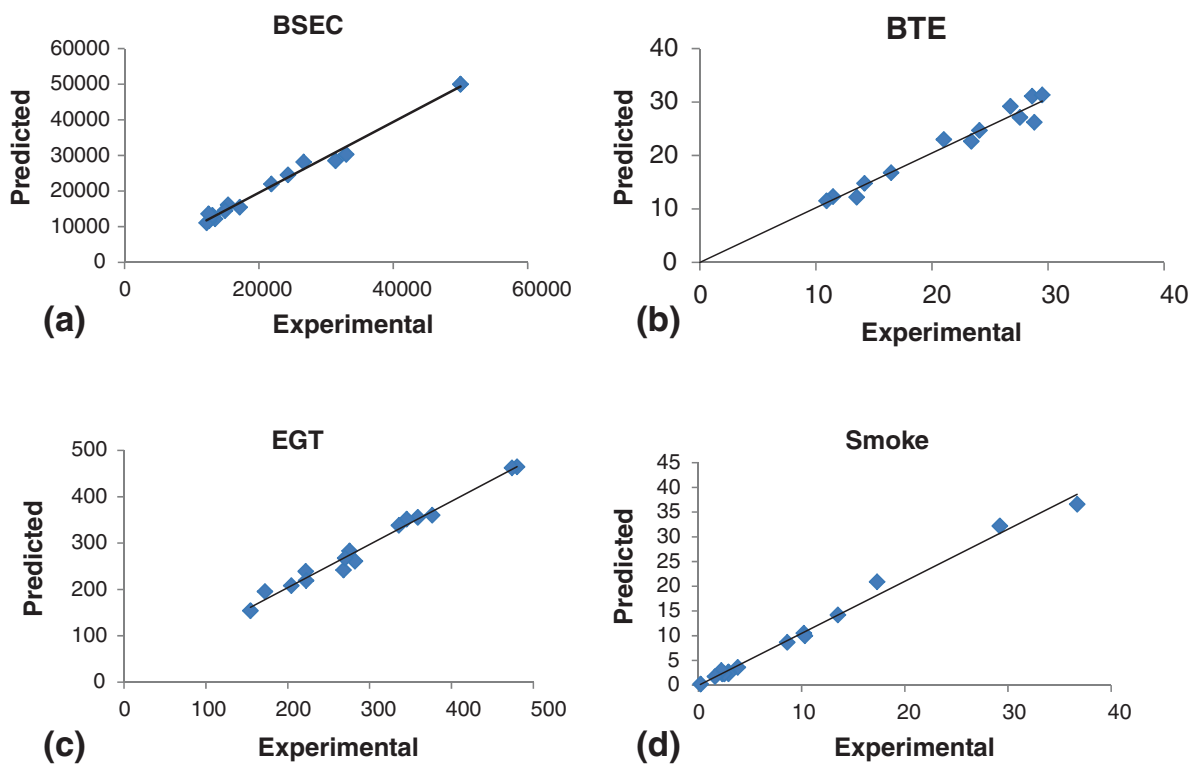

Figure 7. GA Optimized ANFIS Predicted versus Experimental values for (a) BSEC, (b) BTE, (c) EGT and (d) Smoke.

Comparisons of the experimental results and the GA optimized ANFIS predictions demonstrated that LPG-diesel dual fuel engine can be accurately simulated using ANFIS. Figure 7 shows GA optimized ANFIS predicted versus experimental values for BSEC, BTE, EGT and Smoke, respectively. The $\mathrm{R}^{2}$ value and prediction accuracy on test data are given in table 5 . It is clear from the table that GA optimized ANFIS predictions for performance and emission parameters yielded a good statistical performance in terms of values of $\mathrm{R}^{2}$, which are above 0.98 indicating a high level of correlation and prediction accuracy above 90\%. Table 6 to 9 show the GA optimised ANFIS predicted results for all test data (Rai Adarsh 2012).

The $\mathrm{R}^{2}$ value for smoke is slightly higher than for other parameters, when compared to results obtained from ANFIS, but the prediction accuracy on test data $(73.33 \%)$ is much lesser than $90 \%$. This is mainly attributed to smaller difference between the experimental and predicted values in case of smoke than BSEC, for example. Also the calculations of prediction accuracy is based on MRE values, which shows a lot of variation in case of smoke, when compared to BSEC, thereby reducing the prediction accuracy value (Rai Adarsh 2012).

Table 5. $\mathrm{R}^{2}$ and prediction accuracy value for GA Optimized ANFIS model.

\begin{tabular}{lcc}
\hline Parameters & $\mathrm{R}^{2}$ & Prediction accuracy on test data (\%) \\
\hline BSEC & 0.9902 & 100 \\
BTE & 0.9818 & 100 \\
EGT & 0.9833 & 93.33 \\
Smoke & 0.9912 & 73.33 \\
\hline
\end{tabular}


Table 6. Optimal parameters obtained from GA (BSEC).

\begin{tabular}{|c|c|c|c|c|c|c|c|c|}
\hline $\begin{array}{l}\text { Prediction } \\
\text { accuracy }\end{array}$ & $\begin{array}{c}\text { Load } \\
\text { (No. of MF) }\end{array}$ & $\begin{array}{c}\text { IT } \\
\text { (No. of MF) }\end{array}$ & $\begin{array}{c}\% \text { LPG } \\
\text { (No. of MF) }\end{array}$ & $\begin{array}{c}\text { Load } \\
\text { (Type of MF) }\end{array}$ & $\begin{array}{c}\text { IT } \\
\text { (Type of MF) }\end{array}$ & $\begin{array}{c}\text { \%LPG } \\
\text { (Type of MF) }\end{array}$ & $\begin{array}{l}\text { Training Error } \\
\text { (RMSE) }\end{array}$ & Output \\
\hline 100.00 & 5 & 3 & 3 & dsigmf & gaussmf & trimf & $2.88 \mathrm{E}-05$ & linear \\
\hline 100.00 & 5 & 3 & 3 & trimf & gbellmf & pimf & $2.96 \mathrm{E}-05$ & linear \\
\hline 93.33 & 5 & 3 & 3 & gaussmf & gbellmf & gaussmf & 4.69E-05 & linear \\
\hline 93.33 & 5 & 3 & 3 & trimf & gauss $2 \mathrm{mf}$ & gaussmf & $2.87 \mathrm{E}-05$ & linear \\
\hline 93.33 & 5 & 3 & 3 & trimf & gbellmf & gaussmf & $3.05 \mathrm{E}-05$ & linear \\
\hline 93.33 & 5 & 3 & 3 & trimf & dsigmf & trimf & $2.68 \mathrm{E}-05$ & linear \\
\hline 86.67 & 5 & 3 & 3 & gauss $2 \mathrm{mf}$ & dsigmf & gaussmf & $3.94 \mathrm{E}-05$ & linear \\
\hline
\end{tabular}


Table 7. Optimal parameters obtained from GA (BTE).

\begin{tabular}{|c|c|c|c|c|c|c|c|c|}
\hline $\begin{array}{l}\text { Prediction } \\
\text { accuracy }\end{array}$ & $\begin{array}{c}\text { Load } \\
\text { (No. of MF) }\end{array}$ & $\begin{array}{c}\text { IT } \\
\text { (No. of MF) }\end{array}$ & $\begin{array}{c}\% \text { LPG } \\
\text { (No. of MF) }\end{array}$ & $\begin{array}{c}\text { Load } \\
\text { (Type of MF) }\end{array}$ & $\begin{array}{c}\text { IT } \\
\text { (Type of MF) }\end{array}$ & $\begin{array}{c}\% \text { LPG } \\
\text { (Type of MF) }\end{array}$ & $\begin{array}{c}\text { Training } \\
\text { Error (RMSE) }\end{array}$ & Output \\
\hline 100.00 & 5 & 3 & 3 & gbellmf & trapmf & trimf & $5.31 \mathrm{E}-05$ & linear \\
\hline 100.00 & 5 & 3 & 3 & gbellmf & trimf & trimf & $5.50 \mathrm{E}-05$ & linear \\
\hline 100.00 & 5 & 3 & 3 & gbellmf & gauss $2 \mathrm{mf}$ & gbellmf & 7.03E-05 & linear \\
\hline 100.00 & 5 & 3 & 3 & gbellmf & gauss $2 \mathrm{mf}$ & gbellmf & 7.03E-05 & linear \\
\hline 100.00 & 5 & 3 & 3 & gbellmf & trimf & gbellmf & $7.45 \mathrm{E}-05$ & linear \\
\hline 93.33 & 5 & 3 & 3 & gbellmf & gauss $2 \mathrm{mf}$ & psigmf & $5.22 \mathrm{E}-05$ & linear \\
\hline 93.33 & 5 & 3 & 3 & gbellmf & trapmf & psigmf & $5.37 \mathrm{E}-05$ & linear \\
\hline
\end{tabular}


Table 8. Optimal parameters obtained from GA (EGT).

\begin{tabular}{|c|c|c|c|c|c|c|c|c|}
\hline $\begin{array}{l}\text { Prediction } \\
\text { accuracy }\end{array}$ & $\begin{array}{c}\text { Load } \\
\text { (No. of MF) }\end{array}$ & $\begin{array}{c}\text { IT } \\
\text { (No. of MF) }\end{array}$ & $\begin{array}{c}\text { \%LPG } \\
\text { (No. of MF) }\end{array}$ & $\begin{array}{c}\text { Load } \\
\text { (Type of MF) }\end{array}$ & $\begin{array}{c}\text { IT } \\
\text { (Type of MF) }\end{array}$ & $\begin{array}{c}\% \mathrm{LPG} \\
\text { (Type of MF) }\end{array}$ & $\begin{array}{l}\text { Training Error } \\
\text { (RMSE) }\end{array}$ & Output \\
\hline 93.33 & 3 & 3 & 3 & gauss $2 \mathrm{mf}$ & gbellmf & trimf & $2.58 \mathrm{E}-01$ & linear \\
\hline 93.33 & 3 & 4 & 3 & gauss $2 \mathrm{mf}$ & gbellmf & trimf & $3.06 \mathrm{E}-01$ & linear \\
\hline 93.33 & 3 & 4 & 3 & gauss $2 \mathrm{mf}$ & gbellmf & trimf & $3.06 \mathrm{E}-01$ & linear \\
\hline 93.33 & 3 & 4 & 3 & gauss $2 \mathrm{mf}$ & gbellmf & trimf & 3.06E-01 & linear \\
\hline 93.33 & 3 & 4 & 3 & gauss $2 \mathrm{mf}$ & gbellmf & trimf & $3.06 \mathrm{E}-01$ & linear \\
\hline 66.67 & 3 & 4 & 4 & gauss $2 \mathrm{mf}$ & gbellmf & trimf & $6.04 \mathrm{E}-03$ & linear \\
\hline 60.00 & 3 & 3 & 6 & gauss $2 \mathrm{mf}$ & gbellmf & trimf & $1.19 \mathrm{E}-04$ & linear \\
\hline
\end{tabular}


Table 9. Local optimal parameters obtained from GA (Smoke).

\begin{tabular}{|c|c|c|c|c|c|c|c|c|}
\hline $\begin{array}{l}\text { Prediction } \\
\text { accuracy }\end{array}$ & $\begin{array}{c}\text { Load } \\
\text { (No. of MF) }\end{array}$ & $\begin{array}{c}\text { IT } \\
\text { (No. of MF) }\end{array}$ & $\begin{array}{c}\% \text { LPG } \\
\text { (No. of MF) }\end{array}$ & $\begin{array}{c}\text { Load } \\
\text { (Type of MF) }\end{array}$ & $\begin{array}{c}\text { IT } \\
\text { (Type of MF) }\end{array}$ & $\begin{array}{c}\% \mathrm{LPG} \\
\text { (Type of MF) }\end{array}$ & $\begin{array}{l}\text { Training Error } \\
\text { (RMSE) }\end{array}$ & Output \\
\hline 73.33 & 6 & 3 & 3 & gaussmf & gaussmf & trimf & $1.20 \mathrm{E}-04$ & linear \\
\hline 66.67 & 5 & 3 & 3 & gauss $2 \mathrm{mf}$ & trapmf & trimf & $6.69 \mathrm{E}-05$ & linear \\
\hline 66.67 & 5 & 3 & 3 & gauss $2 \mathrm{mf}$ & gauss $2 \mathrm{mf}$ & trimf & $6.81 \mathrm{E}-05$ & linear \\
\hline 60.00 & 5 & 4 & 3 & gaussmf & trimf & trimf & $1.46 \mathrm{E}-04$ & linear \\
\hline 53.33 & 5 & 4 & 3 & gaussmf & gbellmf & trimf & $1.36 \mathrm{E}-04$ & linear \\
\hline 46.67 & 6 & 3 & 3 & gbellmf & trimf & pimf & $8.54 \mathrm{E}-05$ & linear \\
\hline 46.67 & 6 & 3 & 3 & gaussmf & gaussmf & psigmf & $9.94 \mathrm{E}-05$ & linear \\
\hline
\end{tabular}


Table 10. Comparison of ANFIS and GA optimized ANFIS.

\begin{tabular}{|c|c|c|c|c|c|}
\hline \multirow[b]{2}{*}{ Sl. No. } & \multirow[b]{2}{*}{ Parameters } & \multicolumn{2}{|r|}{$\mathrm{R}^{2}$} & \multicolumn{2}{|c|}{ Prediction accuracy (\%) } \\
\hline & & $\overline{\text { ANFIS }}$ & GA Optimized ANFIS & ANFIS & GA Optimized ANFIS \\
\hline 1 & BSEC & 0.986 & 0.9902 & 93.33 & 100 \\
\hline 2 & BTE & 0.977 & 0.9818 & 86.67 & 100 \\
\hline 3 & EGT & 0.983 & 0.9833 & 93.33 & 93.33 \\
\hline 4 & Smoke & 0.885 & 0.9912 & 46.67 & 73.33 \\
\hline
\end{tabular}

\section{Comparison of models}

In the present study, ANFIS and GA Optimized ANFIS have been applied for prediction of performance and emission parameters of an LPG Diesel dual fuel engine. The results of comparison have been presented in table 10 .

A few important observations that can be made are as follows:

(i) ANFIS-based model for predicting performance and emission parameters in a dual fuel engine is effective, with poor prediction accuracy for smoke.

(ii) Use of GA for optimizing the ANFIS modelling performance, drastically improves with $100 \%$ prediction accuracy for performance parameters and slightly poor performance for smoke with $73.33 \%$ prediction accuracy. This is a substantial improvement over ANFIS based model, which is only $46.67 \%$.

(iii) $\mathrm{R}^{2}$ value is not really an effective parameter in evaluating the prediction performance of ANFIS based models. (Rai Adarsh 2012)

Figures 8 to 11 show the plots of experimental, ANFIS and GA Optimized ANFIS. Figure 8 shows for BSEC, the closeness between experimental and predicted values is better, when compared to BTE, where inspite of BTE being the inverse of BSEC, there is some error between experimental and predicted values which is given in figure 9. Figure 10 describes the comparison of model performance for EGT, which shows closeness with experimental values, whereas for figure 11, with respect to smoke, there is considerable drift between the two due to measurement error. The MRE is high and accordingly the prediction accuracy is very low. The measurement process of smoke using the instrument might have contributed to the variation and also the values

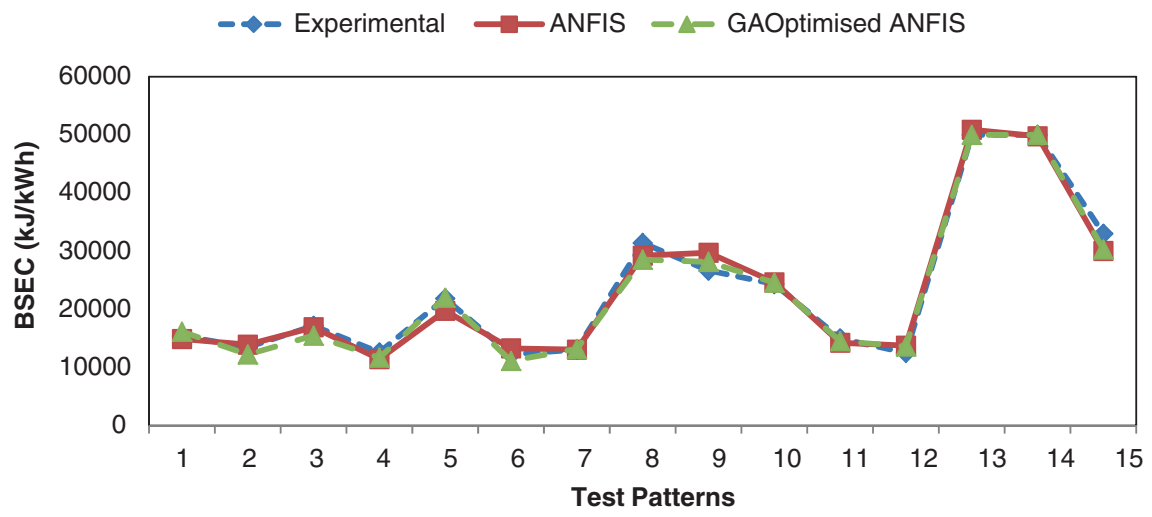

Figure 8. Comparison of Experimental, ANFIS and GA Optimized ANFIS (BSEC). 


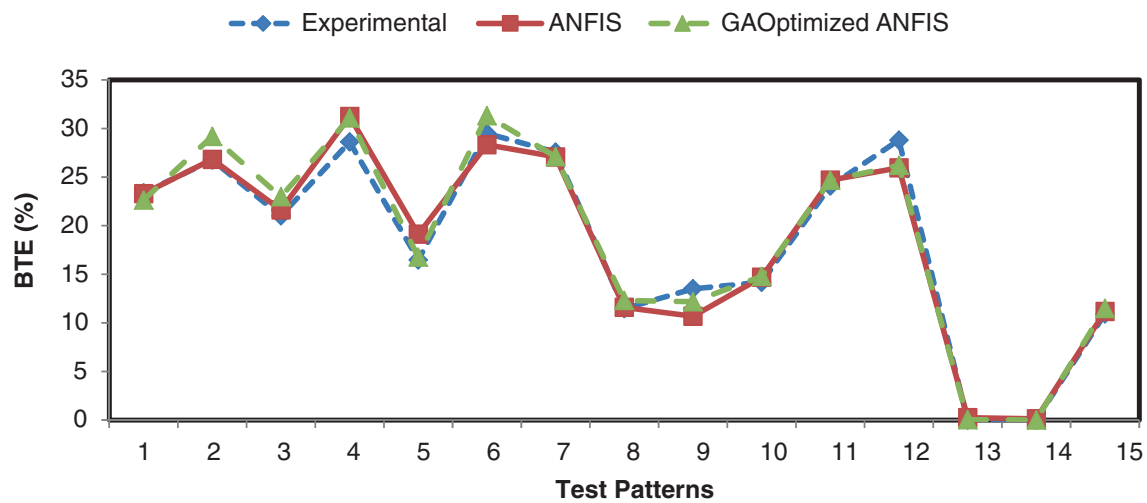

Figure 9. Comparison of Experimental, ANFIS and GA optimized ANFIS (BTE).

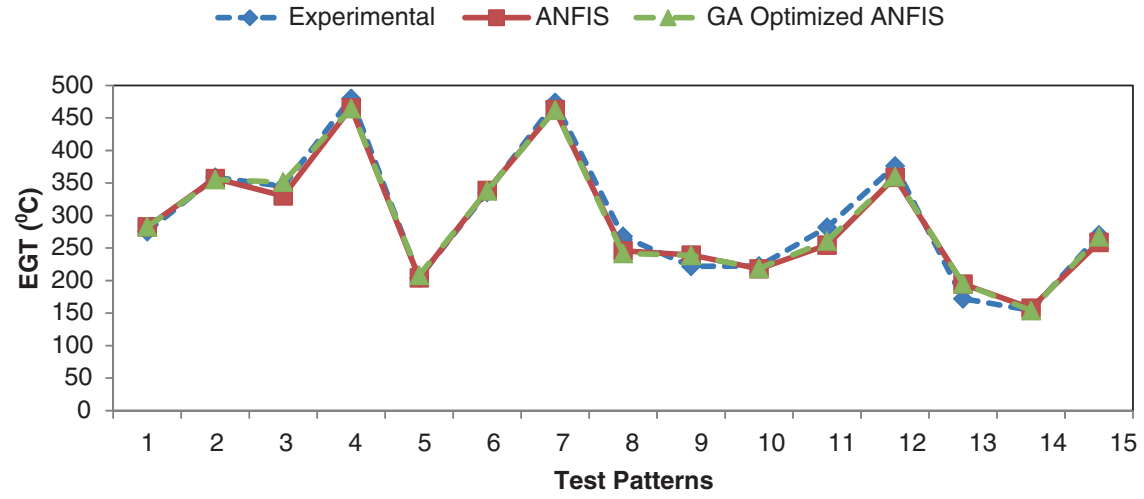

Figure 10. Comparison of Experimental, ANFIS and GA optimized ANFIS (EGT).

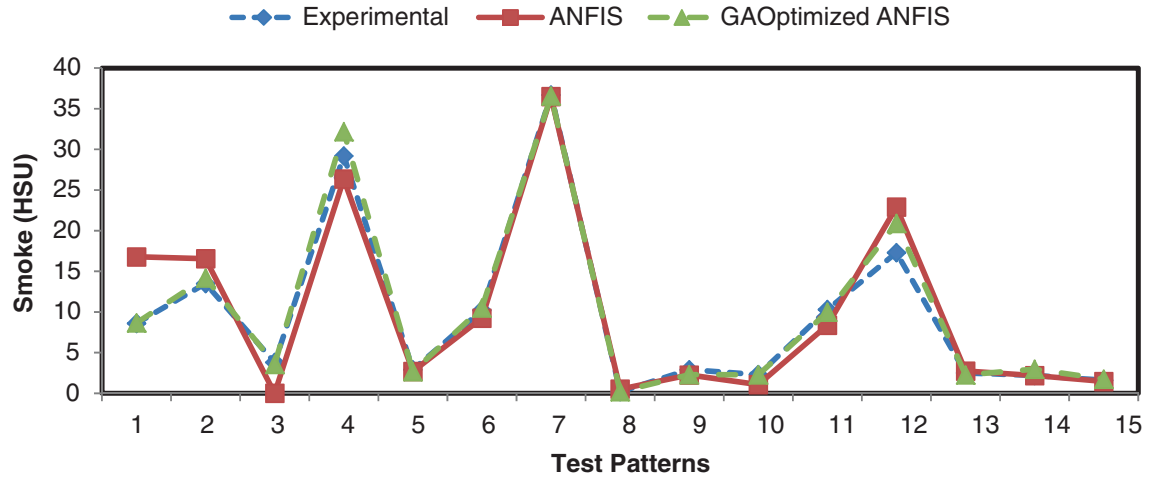

Figure 11. Comparison of Experimental, ANFIS and GA optimized ANFIS (Smoke). 
of smoke undergo a substantial increase with the increase in load percentage. Hence even a small variation will lead to large error values. It is clear from the figures that GA Optimized ANFIS is effective in predicting the values of performance and emission parameters (Rai Adarsh 2012).

\section{Conclusions}

In the present study, LPG-Diesel has been used to run the dual fuel engine under different operating conditions. Experiments were conducted using different percentage of LPG at different loads and at different injection timings. The experimental results obtained were used for modelling the performance parameters and emissions using ANFIS and GA optimized ANFIS. In the previous effort by the authors (Rai et al 2012) an attempt was made to compare the performance of conventional fuzzy logic modelling with ANFIS modelling for the same problem. It was found that ANFIS performed substantially better than conventional fuzzy logic based model.

Further, in this work in order to improve the ANFIS prediction performance, GA has been used to optimize the type and number of membership functions in ANFIS modelling. GA optimized ANFIS provided a prediction accucy of more than $90 \%$ for all parameters, except for smoke for which it has been $73.33 \%$. This is a considerable improvement over ANFIS, which is $46.67 \%$. This establishes the suitability of using GA for optimizing the ANFIS model which has not been widely used in dual fuel engine modelling studies.

\section{References}

Al-Hinti I, Samhouri M, Al-Ghandoor A and Sakhrieh A 2009 The effect of boost pressure on the performance characteristics of a diesel engine: A neuro-fuzzy approach. Applied Energy 86: 113-121

Bailkeri Narasimha 2005 Performance studies on Sunflower Oil Ester-LPG dual-fuel engine. M-Tech Thesis, NMAMIT, Nitte, Visvesvaraya Technological University, Belgaum

Ganesan S and Ramesh A 2002 Experimental Investigations on a LPG-Diesel Dual-Fuel Engine. J. Institution of Engineers 83: 105-111

Hong-Hee Lee, Ngoc-Tu Nguyen and Jeong-Min Kwon 2007 Bearing Fault Diagnosis Using Fuzzy Inference Optimized by Neural Network and Genetic Algorithm. J. Electrical Eng. Technol. 2: 353-357

Jang J S R 1993 ANFIS: Adaptive-network-based fuzzy inference system. IEEE Transactions on Systems, Man, and Cybernetics 23: 665-685

Jang J S, Sun C T and Mizutani E 1997 ANFIS (Adaptive Neuro-Fuzzy Inference Systems), Neuro Fuzzy and Soft Computing-A Computational Approach to Learning and Machine Intelligence. PHI Learning Pvt Ltd, 336-337

Jose Aneesh 2011 Performance Prediction of LPG-Sunflower Oil Ester Dual Fuel C.I Engine using Artificial Neural Networks. M-Tech Thesis, NMAMIT, Nitte, Visvesvaraya Technological University, Belgaum

José M Alonso, Fernando Alvarruiz, José M Desantes, Leonor Hernández, Vicente Hernández and Germán Moltó 2007 Combining Neural Networks and Genetic Algorithms to Predict and Reduce Diesel Engine Emissions. IEEE Transactions on Evolutionary Computation 11: 46-55

Lee S H, Howlett R J, Crua C and Walters S D 2007 Fuzzy logic and neuro-fuzzy modelling of diesel spray penetration: A comparative study. J. Intelligent and fuzzy systems 18: 43-56

MATLAB R 2012 http://www.mathworks.in/help/fuzzy/anfisedit.html

Mohd. Ariffanan Bin Mohd Basri A 2008 Medical Image Classification And Symptoms Detection Using Neuro Fuzzy. Master of Engineering (Electrical - Mechatronics and Automatic Control) Universiti Teknologi Malaysia

Park Sungwook 2012 Optimization of combustion chamber geometry and engine operating conditions for compression ignition engines fuelled with dimethyl ether. Fuel 97: 61-71 
Piccolo A 2001 Optimisation of energy flow management in hybrid electric vehicles via Genetic Algorithms. IEEE transactions on Advanced Intelligent Mechatronics Proceedings 1: 434-439

Poonia M P, Ramesh A and Gaur R R 1999 Experimental Investigation of the Factors Affecting the Performance of a LPG - Diesel Dual-Fuel Engine. SAE Transactions. J. Fuels and Lubricants 499-503

Rai Adarsh A 2012 Fuzzy Logic based Prediction of Performance and Emission Parameters of a Dual Fuel Compression Ignition Engine. M-Tech Thesis, NMAMIT, Nitte, Visvesvaraya Technological University, Belgaum

Rai Adarsh, Kumar Satheesh N, Pai Srinivasa P and Rao Shrinivasa B R 2012 Fuzzy logic based Prediction of Performance and Emission Parameters of a LPG-Diesel dual fuel engine. Procedia Engineering 38: 280-292

Sugeno Michio and Takagi Tomohiro 1983 Multi-dimensional fuzzy reasoning. Fuzzy Sets and Systems 9: 313-325 